\title{
Performance and Stability of an Agile Tail-less MAV with Flexible Articulated Wings
}

\author{
Aditya A. Paranjape*, Animesh Chakravarthy† Soon-Jo Chung $\ddagger$ and Harry H. Hilton ${ }^{\S}$
}

This paper considers the problems of (a) modelling the flight mechanics of a tail-less MAV equipped with flexible articulated wings, and (b) the analysis of its turning performance. The wings are assumed to have two degrees of freedom - heave and twist. They are assumed to be actuated from the root, which is the abstraction of an experimental control mechanism being developed by the authors. The dihedral and twist angles at the wing root are controlled. A novel actuator concept of axial tension to control wing stiffness has been explored in this paper. It is shown that axial tension in the wing has a significant effect on the turning performance of the aircraft, although the effect is not uniformly beneficial in nature. The effect of flexibility on the steady state turning performance of the aircraft has been demonstrated by comparing it with that of a rigid aircraft, and with that of a similar aircraft possessing a wing with different elastic properties.

\section{Nomenclature}

$\begin{array}{cl}D, L, Y & \text { drag, lift and side force } \\ J_{R}, J_{L}, J & \text { moment of inertia tensor of the right and left wings, and the aircraft } \\ m_{w, R}, m_{w, L} & \text { body respectively, in the aircraft body frame } \\ m & \text { total mass of the aircraft } \\ p, q, r & \text { body axis roll, pitch and yaw rates } \\ \mathbf{r}_{C G} & \text { position vector of the aircraft center of gravity } \\ S(p) q & \text { cross product } p \times \text { where } p, q \in \mathbb{R}^{3} \\ T & \text { axial tension in the wing } \\ T_{F G} & \text { rotation matrix from frame } \mathrm{G} \text { to frame } \mathrm{F} \\ \mathbf{u}_{F} & \text { velocity vector with components in frame } \mathrm{F} \\ u, v, w & \text { body axis aircraft wind velocity components } \\ \alpha & \text { angle of attack } \\ \beta & \text { sideslip angle (but see next entry) } \\ \beta_{\{\cdot\}} & \text { backward sweep on a wing (see subscripts) } \\ \gamma & \text { flight path angle } \\ \delta_{\{\cdot\}} & \text { actuator deflection angle (see subscripts) } \\ \theta & \text { twist (also denotes Euler pitch angle and wing twist at the root) } \\ \phi, \theta, \psi & \text { Euler angles } \\ \xi & \text { bending displacement } \\ \omega_{\{\cdot\}} & \text { angular velocity (see subscripts) } \\ & \end{array}$

*Doctoral candidate, Dept. of Aerospace Engineering., Univ. of Illinois at Urbana-Champaign (UIUC), Urbana, IL; Student Member, AIAA; paranja2@illinois.edu

$\dagger$ Assistant Research Professor, Univ. of Florida REEF, Shalimar, FL. Member, AIAA. animesh@reef.ufl.edu.

$¥$ Assistant Professor, Dept. of Aerospace Engineering, UIUC. Senior Member, AIAA; sjchung@illinois.edu

$\S$ Professor Emeritus, Dept. of Aerospace Engineering, UIUC. Fellow, AIAA; h-hilton@illinois.edu 


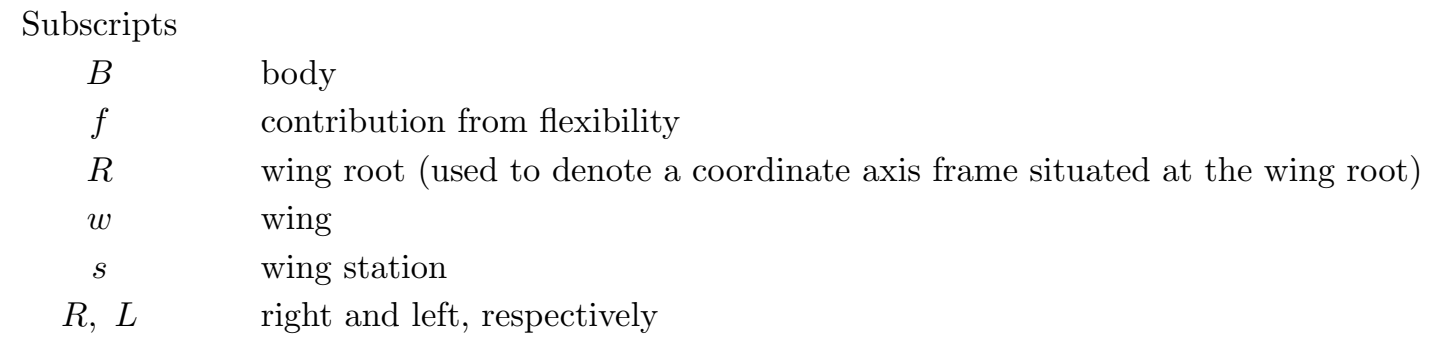

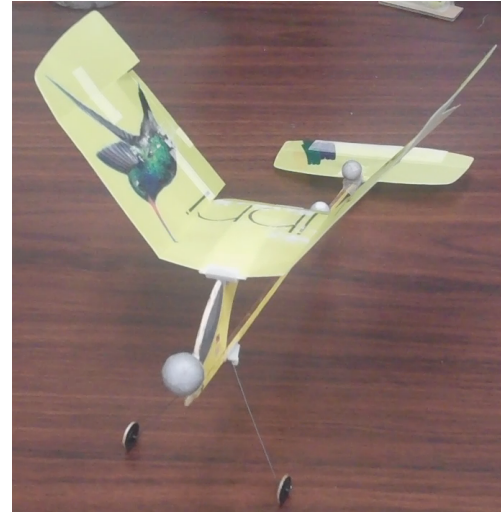

(a) Plantraco Kolibri in a high dihedral configuration during experiments.

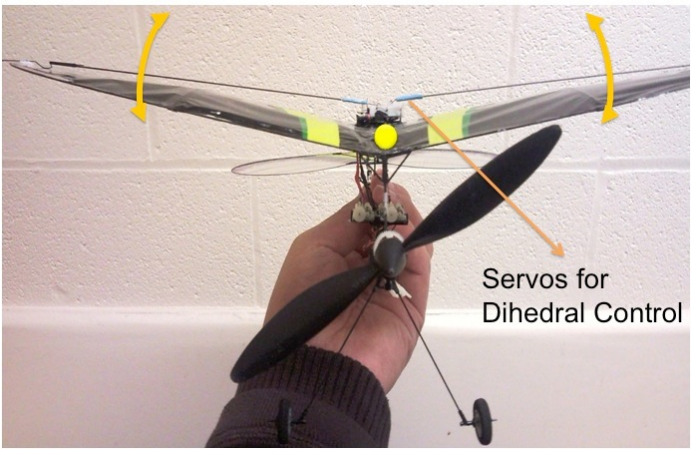

(b) Mechanism for controlling the dihedral angle of the wings.

Figure 1. Aircraft used for initial experiments, and a mechanism to control wing dihedral using wing flexibility in bending.

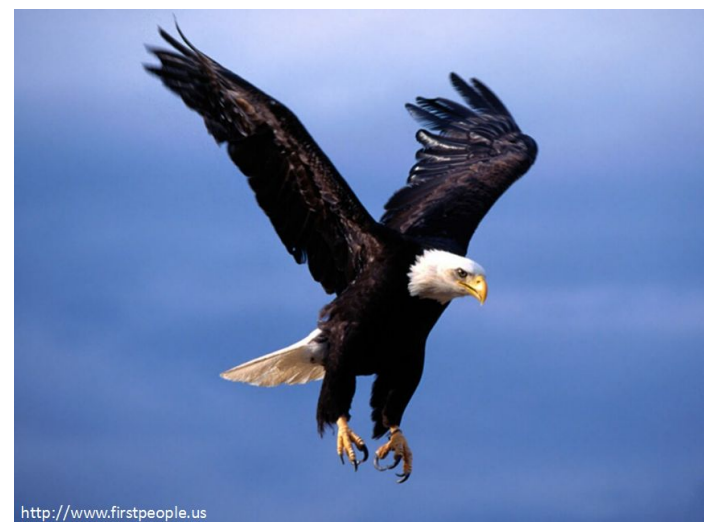

(a) Eagle in descent (source: firstpeople.us)

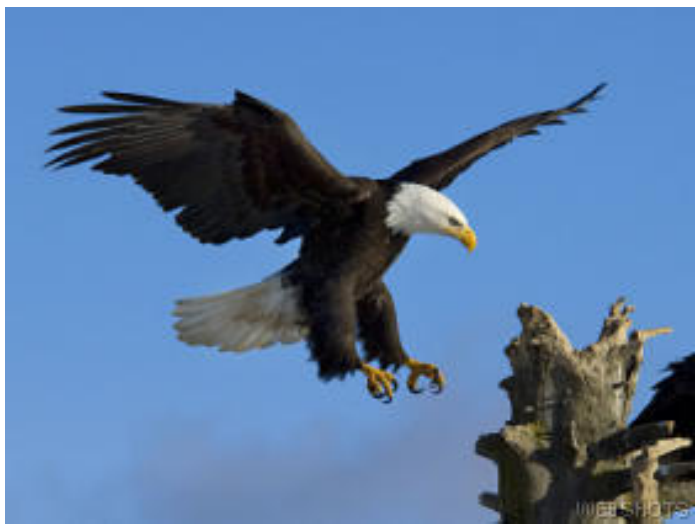

(b) Eagle while perching (source:yimwhan.com)

Figure 2. Eagle during a (steep) descent and during perching.

\section{Introduction}

One of the challenging problems facing the aeronautical community is developing unmanned aerial vehicles which are autonomous, agile and which possess the capability to fly in constrained environments [1]. Birds are ubiquitous examples of such vehicles and therefore, a natural choice for role models for agile micro aerial vehicles (MAVs) wherein these attributes can be engineered. Complex maneuvers require a combination of open and closed loop capabilities. Nevertheless, the performance achievable in the closed loop (with control 
and guidance) is governed and constrained by the capabilities of the airframe. In this spirit, this paper is focussed on some aspects of the open-loop performance of agile MAVs.

In particular, this paper considers a new fixed wing aircraft concept motivated by avian wings. ${ }^{2}$ The focus of this paper is on a flexible wing which can be rotated at the root to generate variable dihedral and twist. The axial tension in the wing can be controlled as well. The dihedral angles on the port and starboard wings need not be equal. This concept of asymmetric dihedral is used for yaw stability and control in the absence of a vertical tail. Fig. 1(a) shows the Plantraco Kolibri MAV with its wing dihedral set to 55 deg for experiments conducted by the Aerospace Robotics and Control Group at the University of Illinois at Urbana-Champaign to study and vaildate the ideas presented in this paper. Fig 1(b) shows a mechanism being designed for an aircraft with a highly flexible wing. Aerodynamic contributions of the fuselage and thrust have been ignored with the understanding that they can be readily added within the conceptual framework of this paper.

\section{A. Literature Review}

Fixed and flapping wing MAVs have been extensively studied in literature. The compendium of papers Ref. [3] is an excellent reference for some of the work done in this area until the turn of the $21^{\text {st }}$ century. Costello and Webb [4] demonstrated that articulated wing MAVs, with wings hinged at the root, could reduce gust sensitivity. Wickenheiser and Garcia $[5,6]$ studied the dynamics of morphing aircraft and demonstrated perching using, among other forms of articulation, variable wing incidence. Reich et al. [7] experimentally studied the aerodynamic performance of a wing of variable incidence for perching. The lateral stability and control of birds, and in particular, the role of wing dihedral, have been studied extensively by Sachs [8-10]. Sachs has demonstrated that for air vehicles whose size and speed are similar to that of birds, wings are sufficient to provide lateral stability, thereby reducing, if not eliminating altogether, the need for a vertical tail. Paranjape and $\mathrm{Chung}^{2}$ demonstrated the utility of asymmetric dihedral for yaw control and the use of symmetric dihedral for longitudinal maneuvers for a rigid aircraft. They also provided analytical approximations to evaluate the lateral stability of tailless aircraft flying at low speeds. ${ }^{11}$ This paper is meant to extend those ideas to a flexible aircraft. The most critical element of that extension is modelling wing flexibility.

A variety of aircraft models incorporating wing and fuselage flexibility have been proposed in literature. Waszak and Schmidt ${ }^{12}$ gave a complete nonlinear, if somewhat simplistic, model of an aircraft with flexible wings. Their aerodynamic model, however, assumed steady flow, and their frame of reference consisted of the so-called mean axes which are hard to locate in a practical situation. Tuzcu and Meirovitch ${ }^{13}$ extended their model in several ways: they used more a intuitive reference frame (the conventional body axes) and a more accurate Theodorsen's theory for modelling the aerodynamics of the wing. Recently, Tuzc and Nguyen ${ }^{14}$ presented a dynamics model for a fully flexible aircraft. These papers worked with a small-strain, smalldisplacement beam theory. In contrast, recently, Patil and co-authors ${ }^{15,16}$ derived a geometrically exact (large displacement) small-strain nonlinear beam model, and used it to study the dynamics and stability of flying wings. Baghdadi and co-authors ${ }^{17}$ used bifurcation analysis to study the performance and stability of a flexible aircraft model based on Ref. [12].

\section{B. Main Contributions}

This paper is intended to contribute towards the broader problem of developing a flapping MAV capable of agile flight in constrained environments. Chung and Dorothy ${ }^{18}$ studied a neurobiologically-inspired controller for flapping flight, and demonstrated it on a robotic testbed. Their controller, similar to avian flight, could switch in a stable and smooth fashion between flapping and gliding flight. Among other phases of flight, gliding is essential during landing. This paper provides the flight mechanic foundations needed to design effective gliding strategies in the presence of flexible wing dynamics. The concept of the effective dihedral of a flexible wing is introduced in the paper. Trim calculations have been performed for steady gliding turns, and the effects of wing flexibility on these trims have been highlighted by comparing the trim plots with those obtained for an identical aircraft with rigid wings. ${ }^{2}$ The effect of the axial tension in the wing on the turning performance has been studied. It has been shown that the axial tension increases the demands on the actuation system, but at least for the particular aircraft model considered here, improves its stability characteristics. 
The dynamic modelling integrates rigid body and flexible wing dynamics. The wings are assumed to be rectangular with uniform elastic properties. The Euler-Bernoulli beam theory has been used to model the wings. All equations are derived with respect to a conveniently chosen body axis frame. For the purpose of modelling wing flexibilty, the wing is assumed to be clamped to the fuselage. The aerodynamic forces are calculated using the model proposed by Dickinson, Lehmann and Sane. ${ }^{19}$

This paper is intended as a first step in solving a much more challenging problem. This paper has several limitations. The aircraft stability calculations are not complete. The structural model of the wing needs to be replaced with one which incorporates large deformations and the accompanying nonlinear effects ${ }^{15,16}$ in keeping with the nature of the problem. A case can be made to model the wing as a membrane which would complicate the aeroelastic calculations considerably. Work is under way to address these shortcomings. Despite these limitations, this paper presents several useful results and a reliable foundation for future work on the problem of flexible, agile MAVs.

This paper is oganized as follows. Section II derives the equations of motion for the aircraft with a flexible wing. Section III introduces the concept of the effective dihedral and describes its role in designing the elastic properties of the wing. The effect of axial tension on the effective dihedral is analysed. Section IV describes the trim analysis for a steady turn, and Section V concludes the paper.

\section{Differential Equations}

\section{A. A word on the notation}

Capital letters are reserved for forces and matrices. Small letters are used for scalars when not in bold, and for vectors when used with bold font. Given a vector $x \in \mathbb{R}^{3}, S(x)$ denotes the cross product operator, i.e., for any vector $x \in \mathbb{R}^{3}, S(x) y \triangleq x \times y$. Similarly, $S^{2}(x) y=S(x)(S(x) y)=x \times(x \times y)$. Given a variable $p(t, y)$, its time derivative is denoted by $\dot{p}(t, y) \triangleq \frac{\partial p(t, y)}{\partial t}$. Its spatial derivative is denoted by $p^{\prime}(t, y) \triangleq \frac{\partial p(t, y)}{\partial y}$. Note that when $p(t, y) \equiv p(t), \dot{p}(t)=\frac{d p(t)}{d t}$.

\section{B. Wing Model}

The wing is modelled as an Euler-Bernoulli beam with two degrees of freedom - heave and twist. The wing is loaded under an axial tension, $T(y)$. Structural damping in the wing has been ignored. All calculations have been performed for the right wing, and they can be readily performed for the left wing as well.

\section{Frames of Reference}

Given frames $F$ and $G$, the matrix $T_{\mathrm{FG}}$ is a rotation matrix which transforms the components of a vector from the $G$ frame to $F$. The body frame, denoted by $B$, is attached to the body with the $x-z$ plane coincident with the aircraft plane of symmetry when the wings are undeflected. The $x$ axis points towards the aircraft nose. The $z$ axis points downwards, and the $y$ is defined to create a right handed coordinate system.

The frame $R_{R}$ is the frame based at the right wing root. Its $x$ axis coincides with the $x$ axis of the $B$ frame and it is related to the $B$ frame via a dihedral rotation $\delta_{R}$ at the wing root followed by a rotation $\theta_{R}$ about the $y$ axis. The $y$ axis points along the wing elastic axis. Thus,

$$
T_{\mathrm{BR}}=\left[\begin{array}{ccc}
1 & 0 & 0 \\
0 & \cos \delta_{R} & \sin \delta_{R} \\
0 & -\sin \delta_{R} & \cos \delta_{R}
\end{array}\right]\left[\begin{array}{ccc}
\cos \theta_{R} & 0 & \sin \theta_{R} \\
0 & 1 & 0 \\
-\sin \theta_{R} & 0 & \cos \theta_{R}
\end{array}\right]
$$

The frame $S_{R} \equiv S_{R}(y)$ is the frame located at a spanwise wing station with origin at the elastic center, and $y$ axis pointing along the elastic axis. The frame $S_{R}$ is related to $R_{R}$ via two rotations: a rotation about the $x$ axis through the strain $\xi^{\prime}(y)$, and a rotation (twist) $\theta(y)$ about the y axis. Thus,

$$
T_{\mathrm{R}_{\mathrm{R}} \mathrm{S}_{\mathrm{R}}}=\left[\begin{array}{ccc}
\cos \theta(y) & 0 & \sin \theta(y) \\
\sin \left(\xi^{\prime}(y)\right) \sin \theta(y) & \cos \left(\xi^{\prime}(y)\right) & -\sin \left(\xi^{\prime}(y)\right) \cos \theta(y) \\
-\cos \left(\xi^{\prime}(y)\right) \sin \theta(y) & \sin \left(\xi^{\prime}(y)\right) & \cos \left(\xi^{\prime}(y)\right) \cos \theta(y)
\end{array}\right]
$$




\section{Relative Position Vectors}

Relative position vectors are needed to calculate the cross products associated with the rotational component of the local velocity at a spanwise location on the wing. In particular, we need the position vector of the spanwise station in the $B$ and $S_{R}$ frames. At a spanwise station, we are interested in calculating the velocity at the three quarter chord point for calculating the aerodynamic forces, and the velocity at the elastic axis for calculations related to flexibility.

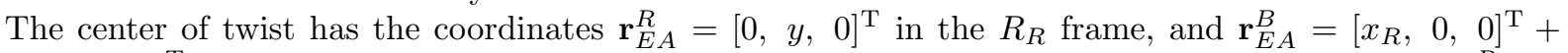
$T_{\mathrm{BR}_{\mathrm{R}}}[0, y, 0]^{\mathrm{T}}$ in the body frame. Likewise, the three quarter chord point has the coordinates $\mathbf{r}_{3 / 4}^{R}=$ $\left[\left(x_{a}-c / 2\right) \cos \theta_{R}(y), y,-\left(x_{a}-c / 2\right) \sin \theta_{R}(y)\right]^{\mathrm{T}}$ in the $R_{R}$ frame, and $\mathbf{r}_{3 / 4}^{B}=\left[\begin{array}{ll}x_{R}, & 0,0\end{array}\right]^{\mathrm{T}}+T_{\mathrm{BR}_{\mathrm{R}}}\left[c\left(x_{a}-\right.\right.$ $\left.1 / 2) \cos \theta_{R}(y), y,-c\left(x_{a}-1 / 2\right) \sin \theta_{R}(y)\right]^{\mathrm{T}}$ in the body frame. Here, $x_{a}$ denotes the chordwise location of the aerodynamic center with respect to the $R_{R}$ frame.

\section{E. Calculating the Velocity at a Spanwise Station}

The velocity at a spanwise location consists of two components - the translational component and the rotational component. The total velocity of the elastic axis, with components in the $S_{R}$ frame, is given by

$$
\mathbf{v}_{E A}(y)=T_{\mathrm{BR}_{\mathrm{R}}}^{\mathrm{T}} T_{\mathrm{R}_{\mathrm{R}} \mathrm{S}_{\mathrm{R}}}^{\mathrm{T}}\left(\left[\begin{array}{c}
u \\
v \\
w
\end{array}\right]+\left[\begin{array}{c}
p \\
q \\
r
\end{array}\right] \times \mathbf{r}_{E A}^{B}\right)+\left[\begin{array}{c}
0 \\
0 \\
\dot{w}(y)
\end{array}\right]+T_{R_{R} S_{R}}^{\mathrm{T}}\left(\left[\begin{array}{c}
-\dot{\delta}_{R} \cos \theta_{R}(y) \\
0 \\
-\dot{\delta}_{R} \sin \theta_{R}(y)
\end{array}\right] \times \mathbf{r}_{E A}^{R}\right)
$$

The total velocity of the three quarter chord point is given by

$$
\mathbf{v}_{3 / 4}(y)=\mathbf{v}_{E A}(y)+\left[\begin{array}{c}
0 \\
0 \\
c\left(x_{a}-1 / 2\right) \dot{\theta}_{R}
\end{array}\right]
$$

Let $\mathbf{v}_{3 / 4}(y)=\left[u_{3 / 4}(y), v_{3 / 4}(y), w_{3 / 4}(y)\right]^{\mathrm{T}}$. Then, the angle of attack and side slip at the section are given by

$$
\alpha(y)=\tan ^{-1}\left(\frac{w_{3 / 4}}{u_{3 / 4}}\right), \quad \beta(y)=\sin ^{-1}\left(\frac{v_{3 / 4}}{\left\|\mathbf{v}_{3 / 4}\right\|}\right)
$$

One can now calculate the aerodynamic forces at the aerodynamic center.

The derivation of the equations of motion can be done by using Newton's laws, or using Hamilton's approach. The former is more convenient for the equations of motion of the aircraft. On the other hand, the latter approach is more convenient for modelling the structural dynamics of the wings.

\section{F. Aircraft Equations of Motion}

Let $m_{F}, m_{R}, m_{L}$ be the masses of the fuselage, the right wing and the left wing, respectively. Let $\tilde{m}_{R}$ and $\tilde{m}_{L}$ denote the masses per unit length of the right wing and the left wing, respectively. Let $\mathbf{r}_{\mathbf{C G}}$ denote the position of the center of gravity of the aircraft. Let $\mathbf{r}_{s}=\left[x_{e} c, 0,0\right]^{\mid r m T}$ denote location of the center of gravity with respect to the wing twist, and let $\mathbf{y}=\left[x_{e} c \cos \theta(y), y, \xi-x_{e} c \sin \theta(y)\right]^{\mathrm{T}}$ denote the position of the center of gravity of a wing station in the wing root frame. Let $\omega_{s} \triangleq\left[\begin{array}{lll}0 & \dot{\theta} & 0\end{array}\right]^{\mathrm{T}}$ denote the angular velocity of a given wing station due twisting. The total linear momentum of the aircraft is the sum of the momenta of the fuselage and the two wings. The momentum and force vectors are written with respect to the body axes, fixed at the wing root. The linear momentum of the aircraft is given by

$$
\begin{aligned}
& \mathbf{p}=m\left(\mathbf{u}_{B}+S\left(\omega_{B}\right) \mathbf{r}_{C G}\right)+\tilde{m}_{R} \int_{0}^{b / 2}\left(S\left(\omega_{R}\right) T_{B R} \mathbf{y}\right) d y+\tilde{m}_{L} \int_{-b / 2}^{0}\left(S\left(\omega_{L}\right) T_{B R} \mathbf{y}\right) d y \\
& +\tilde{m}_{R} \int_{0}^{b / 2}\left(T_{B S} \mathbf{u}_{f, R}(y)+T_{B S} S\left(\omega_{s}\right) \mathbf{r}_{s}\right) d y+\tilde{m}_{L} \int_{-b / 2}^{0}\left(T_{B S} \mathbf{u}_{f, L}(y)+T_{B S} S\left(\omega_{s}\right) \mathbf{r}_{s}\right) d y
\end{aligned}
$$


Differentiating the right hand side with time, and setting $\left[\frac{d \mathbf{p}}{d t}\right]_{I}=\mathbf{F}_{b}$, we get

$$
\begin{aligned}
& m\left(\dot{\mathbf{u}}_{\mathrm{B}}+S(\omega) \mathbf{u}_{B}+S^{2}\left(\omega_{B}\right) \mathbf{r}_{C G}+S\left(\omega_{B}\right) \dot{\mathbf{r}}_{C G}\right)+ \\
& \tilde{m}_{R} \int_{0}^{b / 2}\left(\left(S\left(\omega_{R}+\omega_{B}\right) S\left(\omega_{R}\right)+S\left(\dot{\omega}_{R}\right)\right) T_{B R} \mathbf{y}+S\left(\omega_{R}\right) T_{B S}\left(\omega_{s} \times \mathbf{r}_{s}\right)\right) d y \\
& +\tilde{m}_{L} \int_{-b / 2}^{0}\left(\left(S\left(\omega_{L}+\omega_{B}\right) S\left(\omega_{L}\right)+S\left(\dot{\omega}_{L}\right)\right) T_{B R} \mathbf{y}+S\left(\omega_{R}\right) T_{B S}\left(\omega_{s} \times \mathbf{r}_{s}\right)\right) d y \\
& +\tilde{m}_{R} \int_{0}^{b / 2}\left(T_{B S} \dot{\mathbf{u}}_{f, R}(y)+S\left(\omega_{B}+\omega_{R}+T_{B S} \omega_{s}\right) T_{B S}\left(\mathbf{u}_{f, R}+\omega_{s} \times \mathbf{r}_{s}\right)+T_{B S}\left(\dot{\omega}_{s} \times \mathbf{r}_{s}\right)\right) d y \\
& +\tilde{m}_{L} \int_{-b / 2}^{0}\left(T_{B S} \dot{\mathbf{u}}_{f, L}(y)+S\left(\omega_{B}+\omega_{R}+T_{B S} \omega_{s}\right) T_{B S}\left(\mathbf{u}_{f, L}+\omega_{s} \times \mathbf{r}_{s}\right)+T_{B S}\left(\dot{\omega}_{s} \times \mathbf{r}_{s}\right)\right) d y=\left[\begin{array}{ll}
X Z]^{\mathrm{T}}
\end{array}\right.
\end{aligned}
$$

In the limiting case where $\mathbf{u}_{f}=0$ (i.e, the wings are rigid), the above equation reduces to the corresponding equation in Ref. [2].

Let $I_{p}(y)$ denote the second moment of area matrix. Then, the total angular momentum of the aircraft is given by

$$
\begin{aligned}
& \mathbf{h}=J \omega_{B}+m S\left(\mathbf{r}_{C G}\right) \mathbf{u}_{B}+J_{R} \omega_{R, R}+J_{L} \omega_{R, L}+\tilde{m}_{R} \int_{0}^{b / 2}\left(S\left(T_{B R, R} \mathbf{y}\right) \mathbf{u}_{f}+T_{B S} I_{p}(y)\left(\omega_{B}+\omega_{R, R}+\omega_{s}(y)\right) d y\right. \\
& +\tilde{m}_{L} \int_{-b / 2}^{0}\left(S\left(T_{B R, L} \mathbf{y}\right) \mathbf{u}_{f}+T_{B S} I_{p}(y)\left(\omega_{B}+\omega_{R, L}+\omega_{s}(y)\right) d y,\right.
\end{aligned}
$$

The dynamical equations for rotational motion can be derived from Newton's laws:

$$
\begin{aligned}
& J \dot{\omega}_{B}+S\left(\omega_{B}\right) J \omega_{B}+m S\left(\omega_{B}\right) S\left(\mathbf{r}_{C G}\right) \mathbf{u}_{B}+m S\left(\mathbf{r}_{C G}\right) \dot{\mathbf{u}}_{B}+J_{R} \dot{\omega}_{R_{R}}+J_{L} \dot{\omega}_{R_{L}} \\
& +S\left(\omega_{R}+\omega_{B}\right) J_{R} \omega_{R_{R}}+S\left(\omega_{R_{L}}+\omega_{B}\right) J_{L} \omega_{R_{L}}+S\left(\omega_{R_{R}}\right) J_{R} \omega_{B}+S\left(\omega_{R_{L}}\right) J_{L} \omega_{B}+ \\
& \tilde{m}_{R} \int_{0}^{b / 2}\left(T_{B S} I_{p}\left(\dot{\omega}_{B}+\dot{\omega}_{R, R}+\dot{\omega}_{s, R}(y)\right)+S\left(\omega_{B}+\omega_{R, R}\right) T_{B S} I_{p}\left(\omega_{B}+\omega_{R, R}+\omega_{s, R}\right)\right) d y \\
& +\tilde{m}_{L} \int_{-b / 2}^{0}\left(T_{B S} I_{p}\left(\dot{\omega}_{B}+\dot{\omega}_{R, L}+\dot{\omega}_{s, L}(y)\right)+S\left(\omega_{B}+\omega_{R, L}\right) T_{B S} I_{p}\left(\omega_{B}+\omega_{R, L}+\omega_{s, L}\right) d y\right. \\
& \int_{0}^{b / 2}\left(\tilde{m}_{R}\left(S\left(\omega_{B}+\omega_{R}\right) S\left(T_{B R, R} \mathbf{y}\right) T_{B S} \mathbf{u}_{f, R}+S\left(\omega_{R}\right) S\left(T_{B R, R} \mathbf{y}\right) \mathbf{u}_{B}\right) d y+\int_{0}^{b / 2} \tilde{m}_{R} S\left(T_{B R, R} \mathbf{y}\right) T_{B S} \dot{\mathbf{u}}_{f_{R}} d y\right. \\
& +\int_{-b / 2}^{0}\left(\tilde{m}_{L}\left(S\left(\omega_{B}+\omega_{R_{L}}\right) S\left(T_{B R, L} \mathbf{y}\right) T_{B S} \mathbf{u}_{f, L}+S\left(\omega_{R_{L}}\right) S\left(T_{B R, L} \mathbf{y}\right) \mathbf{u}_{B}\right) d y+\int_{-b / 2}^{0} \tilde{m}_{L} S\left(T_{B R, L} \mathbf{y}\right) T_{B S} \dot{\mathbf{u}}_{f_{L}} d y\right. \\
& +\int_{0}^{b / 2} \tilde{m}_{R} S\left(T_{B R, R} \mathbf{y}\right) S\left(\omega_{s, R}\right) T_{B S} \mathbf{u}_{f_{R}} d y+\int_{-b / 2}^{0} \tilde{m}_{L} S\left(T_{B R, L} \mathbf{y}\right) S\left(\omega_{s, L}\right) T_{B S} \mathbf{u}_{f_{L}} d y=[L M N]^{\mathrm{T}}
\end{aligned}
$$

Note that if flexible terms are ignored along with the wing root angular velocity terms, then, with the additional assumption that $\mathbf{r}_{C G}=0$, Euler's equations are recovered as one would expect.

\section{G. Structural Dynamics}

The equations of structural dynamics of the wing can be derived readily using the formulation for the complete body, with the addition of elastic forces. The bending and twisting equations of motion for the right wing are given by

$$
\left[\begin{array}{cc}
\tilde{m}_{R} & -\tilde{m}_{R} x_{e} c \\
-\tilde{m}_{R} x_{e} c & I_{p}
\end{array}\right]\left[\begin{array}{c}
{\left[\frac{d}{d t}\left(T_{S B} \mathbf{u}_{B}+\mathbf{u}_{f}(y)\right)\right]_{I, 3}} \\
\ddot{\theta}
\end{array}\right]+\left[\begin{array}{cc}
\left(E I \xi^{\prime \prime}\right)^{\prime \prime}-T^{\prime} \xi^{\prime}-T \xi^{\prime \prime} & 0 \\
0 & -G J \theta^{\prime \prime}
\end{array}\right]=\left[\begin{array}{c}
\mathbf{F}_{s, 3} \\
\mathbf{M}_{s, 2}
\end{array}\right]
$$

Here, $\mathbf{F}_{s, 3}$ is the total force acting in the local $z$ direction (hence the subscripts 's' and ' 3 '), while $\mathbf{M}_{s, 2}$ is the local pitching moment. Furthermore,

$$
\left[\frac{d}{d t}\left(T_{S B} \mathbf{u}_{B}+\mathbf{u}_{f}(y)\right)\right]_{I}=T_{S B} \dot{\mathbf{u}}_{B}+\dot{\mathbf{u}}_{f}+S\left(\omega_{B}+\omega_{R}\right)\left(T_{S B} \mathbf{u}_{B}+\mathbf{u}_{f}\right)
$$


and the subscript 3 indicates its third (z-) component. As a simplification, assuming that $T_{S B}=T_{S B}\left(\delta_{R}, \xi^{\prime}\right)$, it can be shown that

$$
\begin{aligned}
{\left[\frac{d}{d t}\left(T_{S B} \mathbf{u}_{B}+\mathbf{u}_{f}(y)\right)\right]_{3}=} & \dot{w} \cos \left(\delta_{R}-\xi^{\prime}\right)+\dot{v} \sin \left(\delta_{R}-\xi^{\prime}\right)+\left(p-\dot{\delta}_{R}+\dot{\xi}^{\prime}\right)\left(\cos \left(\delta_{R}-\xi^{\prime}\right) v-\sin \left(\delta_{R}-\xi^{\prime}\right) w\right) \\
& -(q+\dot{\theta}) u+\ddot{\xi} .
\end{aligned}
$$

The boundary conditions are:

- At the wing root: $\xi=0$, while $\xi^{\prime}$ and $\theta$ can be set arbitrarily (within admissible limits) as the dihedral angle and the twist, respectively, at the wing root.

- At the wing tip: $\xi^{\prime \prime}=0,\left(E I \xi^{\prime \prime}\right)^{\prime}-T \xi^{\prime}=0$ and $\theta^{\prime}=0$ (i.e., free end boundary conditions).

\section{H. Fuselage Kinematics}

The fuselage attitude is described by the Euler angles $\psi, \theta, \phi$. The kinematic equations are given by

$$
\begin{aligned}
\dot{\phi} & =p+q \tan \theta \sin \phi+r \tan \theta \cos \phi, \\
\dot{\theta} & =q \cos \phi-r \sin \phi, \\
\dot{\psi} & =(q \sin \phi+r \cos \phi) \sec \theta
\end{aligned}
$$

\section{Forces}

The forces $\left[\begin{array}{lll}X & Y & Z\end{array}\right]$ consist of two primary components - aerodynamic and gravitational. The aerodynamic forces and moments are computed using strip theory. The wing is divided into chord-wise strips, and using the angle of attack determined locally, the aerodynamic forces are computed using the model in Ref. [19]. They are then transformed to the body frame, which determines $X, Y$ and $Z$. The moments are computed readily.

From Eq. (5), recall that the angle of attack used for computing the aerodynamic forces is given by

$$
\alpha(y)=\tan ^{-1}\left(\frac{w_{3 / 4}}{u_{3 / 4}}\right)
$$

The lift, $L$ and drag $D$ are computed in the local wing frame using the aerodynamic data from Ref. [19]:

$$
C_{l}=0.225+1.58 \sin (2.13 \alpha-7.20), C_{d}=1.92-1.55 \cos (2.04 \alpha-9.82),
$$

where $\alpha$ has been measured in degrees. The local aerodynamic force can be written in the body axis components

$$
\left[\begin{array}{c}
X_{B}(y) \\
Y_{B}(y) \\
Z_{B}(y)
\end{array}\right]=T_{B S}\left[\begin{array}{c}
L(y) \sin \alpha(y)-D(y) \cos \alpha(y) \\
0 \\
-L(y) \cos \alpha(y)-D(y) \sin \alpha(y)
\end{array}\right]
$$

The resulting aerodynamic moment is given by

$$
\left[\begin{array}{c}
L(y) \\
M(y) \\
N(y)
\end{array}\right]=S(\mathbf{r}(y))\left[\begin{array}{c}
X_{B}(y) \\
Y_{B}(y) \\
Z_{B}(y)
\end{array}\right]
$$

where

$$
\mathbf{r}(y)=T_{B R}\left[\begin{array}{l}
0 \\
y \\
0
\end{array}\right]+T_{B S}\left[\begin{array}{c}
0 \\
0 \\
\xi(y)
\end{array}\right]
$$


The gravitational force and moment contributions are given by

$$
\begin{aligned}
& X_{g}=-m g \sin \theta, \quad Y_{g}=m g \cos \theta \sin \phi, \quad Z_{g}=m g \cos \theta \cos \phi, \\
& {\left[\begin{array}{c}
L \\
M \\
N
\end{array}\right]=S\left(\mathbf{r}_{c g}\right)\left[\begin{array}{c}
X_{g} \\
Y_{g} \\
Z_{g}
\end{array}\right]}
\end{aligned}
$$

This completes the formulation of the equations of motion.

\section{J. Trim Equations}

Note that the rigid body equations of motion and the structural dynamic equations are coupled because of acceleration terms. Therefore, for the purpose of locating equilibrium flight conditions (or trims), the rigid body equations of motion and the structural dynamic equations can be decoupled. Specifically, the structural dynamic equations themselves split into bending and twisting equations, which give rise to boundary value problems.

Trims are computed for the flexible-winged aircraft using the fsolve routine in MATLAB. The structural mechanic boundary value problem is solved in- the-loop using MATLAB's built-in boundary value problem solver called bvp4c. ${ }^{20}$ Work is under way to calculate the stability characteristics of the aircraft. In order to determine stability, the structural dynamic equations are converted into a set of ordinary differential equations using Galerkin's method. The resulting set of differential equations, which also includes the equations of motion of the aircraft, are now analysed using fsolve which locates the trim points and their stability.

\section{Analysis of the Wing and Effective Dihedral}

It is instructive to analyse the wing before choosing a value for the Young's modulus, $E$. In a sense, Young's modulus may be considered as a design parameter which can be chosen based on feedback from a flight mechanic analysis such as the one presented in the forthcoming section. In order to exploit the idea of using the wing dihedral for yaw control, the wing dihedral effect itself may be looked upon as one of the design drivers.

The role of differential (or asymmetric) dihedral for yaw control has been discussed in detail in Ref. [2]. The dihedral primarily produces a side force, which is actually a component of the total force produced by the wing normal to its local plane. Let $Y_{B}$ and $Z_{B}$ denote the forces produced by the wing along the body $y$ and $z$ axes, respectively. Therefore, one may define a term called the effective dihedral, $\delta_{\text {eff }}$, as follows:

$$
\delta_{\text {eff }}=\tan ^{-1}\left(\frac{Y_{B}}{Z_{B}}\right)
$$

The notion of effective dihedral is particularly useful for wing design from the point of view of elasticity. The choice of the Young's modulus, E, could be driven by the requirement that the wing produce a sufficient effective dihedral effect with reasonable actuator forces. The effective dihedral depends on the boundary conditions to which the wing is subjected whereas the boundary conditions themselves depend on the location and type of actuators. For a rigid wing, the effective dihedral and the actual dihedral are equal.

As an illustration, consider the following two cases. In the first case, the bending displacement and twist at the wing tip are controlled while the wing root is clamped to the fuselage. In the second case, the wing tip is free while the dihedral (i.e., the bending slope) and twist at the wing root are controlled. The latter scenario would be expected in a flapping wing aircraft, while the former has been partially described in Ref. [22].

Figures 3(a) and 3(b) show the effective wing dihedral for different values of wing tip twist when the actuators produce a given wing displacement or apply a tip load, respectively. This plots demonstrate what might seem intuitive: that the dihedral effect depends largely on the bending profile of the wing, which in turn depends largely on the displacement boundary conditions. This is not altogether obvious because the wing bending is also influenced by twist. The force applied at the wing tip ensures that the net shear force at the tip is zero. Part of the shear force is compensated by the aerodynamic force produced by the wing. In reality, the aerodynamic force at the wing tip is zero and therefore, the three curves in Fig. 3(b) would be expected to merge into a single curve. 


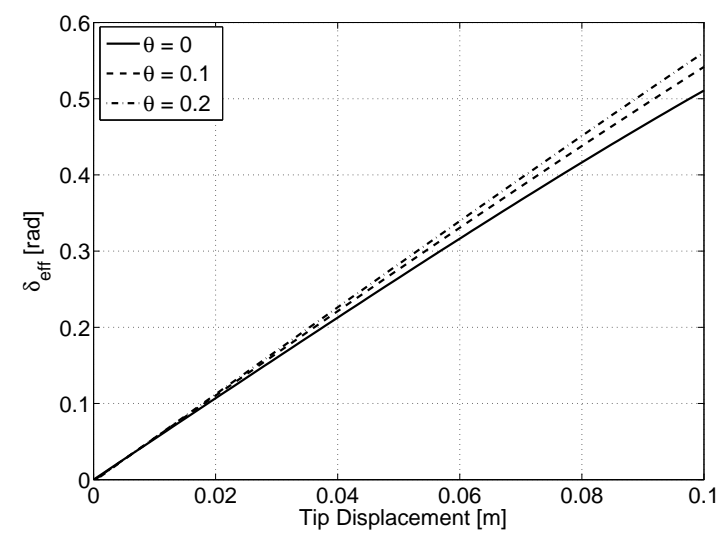

(a) Effective dihedral as a function of tip displacement.

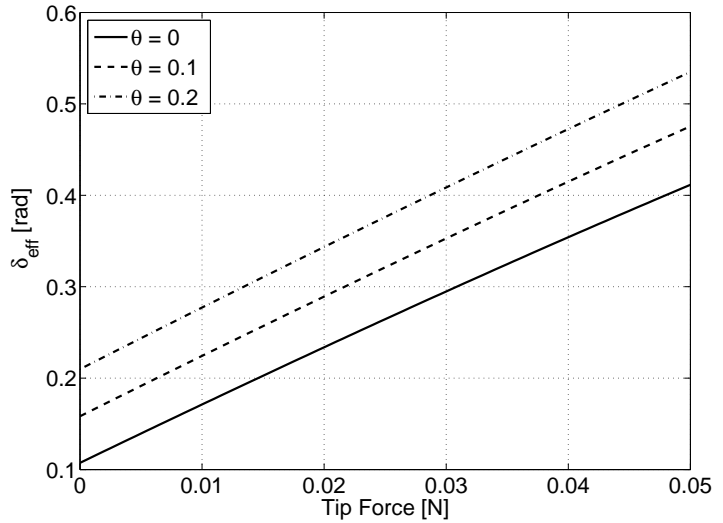

(b) Effective dihedral as a function of the tip force.

Figure 3. Effective dihedral as a function of wing tip displacement and wing tip force, respectively. Each plot shows the effective dihedral for three values of wing tip twist: $0,0.1 \mathrm{rad}$, and $0.2 \mathrm{rad}$.

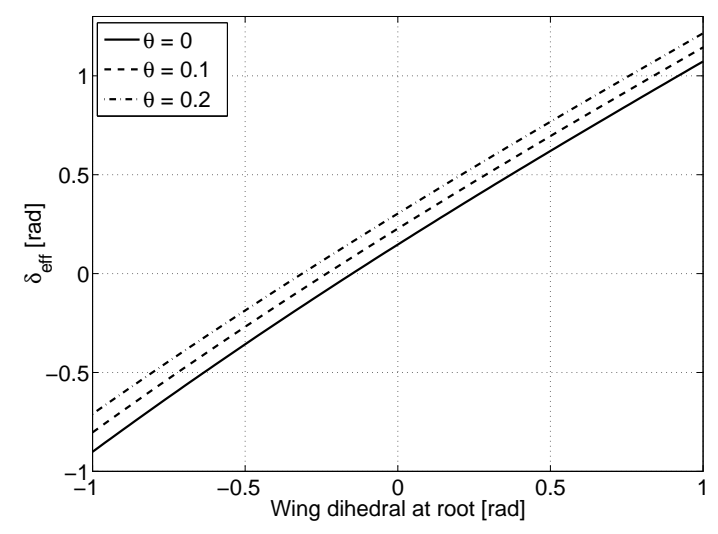

(a) Effective dihedral when $E \sim 1 \mathrm{MPa}$.

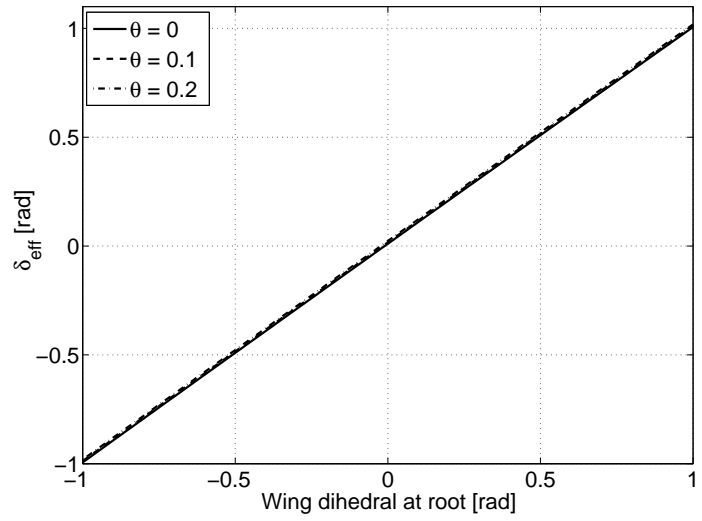

(b) Effective dihedral when $E \sim 10 \mathrm{MPa}$.

Figure 4. Effective dihedral as a function of the dihedral angle at the wing root for two different values of the Young's modulus. Each plot shows the effective dihedral for three values of wing tip twist: $0,0.1$ rad and $0.2 \mathrm{rad}$.

Figures 4(a) and 4(b) show the effective dihedral as a function of the wing dihedral angle at the root. The effective dihedral, as expected, is much higher for $E=5 \mathrm{MPa}$ as compared to $E=50 \mathrm{MPa}$. In the former case, the wing bending is large enough so that the flexibility provides a substantial increase in the wing dihedral effect. This suggests that for the particular wing geometry considered in this paper, a material with a Young's modulus of $E \sim 1 \mathrm{MPa}$ should be chosen in order to obtain a significant dihedral effect. In practice, such analysis should be performed on a case-by-case basis.

At this point, it is helpful to note a design trade-off. A smaller $E$ would provide a larger dihedral effect due to the aerodynamic loads on the wing. However, the same wing would be unable to generate as much anhedral because, usually, the wing would be expected to generate an upward lifting force. This can cause problems for turning flight, as illustrated in the forthcoming section on turning flight. In principle, it seems that this can be alleviated by tautening the wing internally so that it becomes stiffer. The effect of tautening the wing on its effective dihedral effect has been demonstrated in Fig. 5(a). The three curves in the figure correspond to tensions of zero, 5 g., and $10 \mathrm{~g}$., respectively. The Young's modulus was set to $E=5 \mathrm{MPa}$. The tension values were chosen to be commensurate with the weight of the aircraft, with the understanding that servos similar to those which maneuver the wing should be able to provide these values of tension. Clearly, 


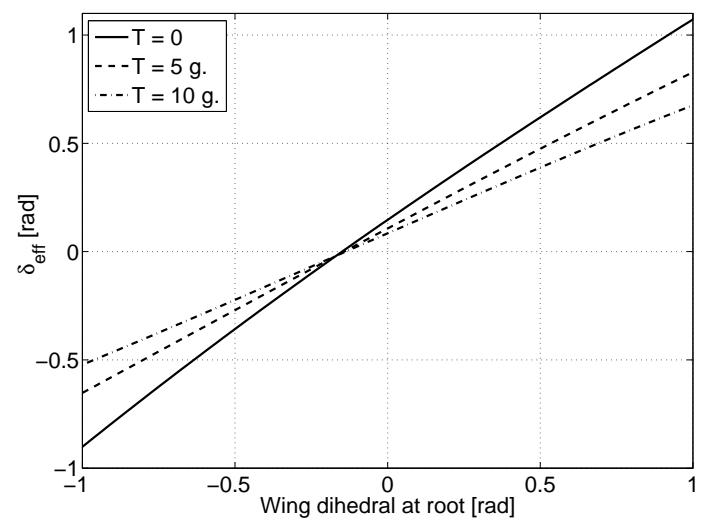

(a) Effective dihedral when $E \sim \mathrm{MPa}$.

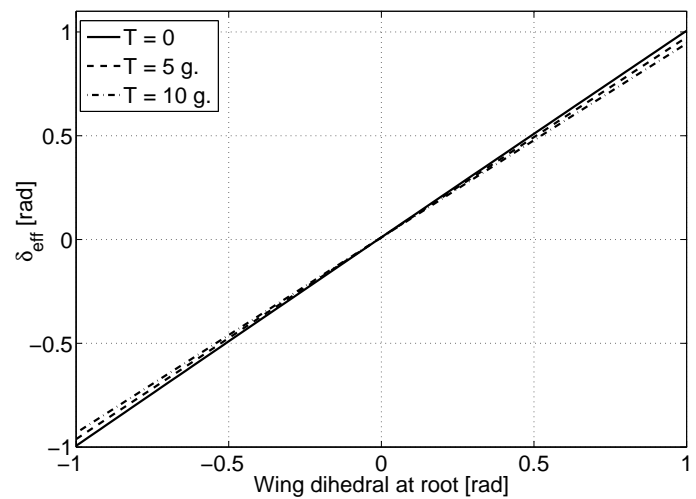

(b) Effective dihedral when $E \sim 10 \mathrm{MPa}$.

Figure 5. Effect of tension on the effective dihedral. The curves corresponding to a tension of a zero, $5 \mathrm{~g}$., and 10 g. are plotted.

the effective dihedral decreases substantially with tension. The effect of tension becomes less significant as the Young's modulus of the material is increased, as shown in Fig. 5(b). Whereas the conclusion is quite obvious, such analysis helps choose a suitable Young's modulus for the wing.

Interestingly, tautening the wing not only reduces the effective dihedral of the wing, but it also flattens the curve of the effective dihedral as a function of the dihedral at the wing root. Consequently, when a certain anhedral is required, the tensed wing will produce a lesser anhedral as well! This runs counter to the earlier suggestion of using wing tension to ease the anhedral required at the wing root during a turn. Whether this is purely an outcome of the assumption of a linear structural deformation needs further investigation.

\section{Trim Analysis of Turning Flight}

Once the concept of effective dihedral is understood, one can readily analyse the performance of the aircraft during steady maneuvers in a manner similar to that described for a rigid aircraft in Ref. [2]. In fact, it turns out that longitudinal flight performance of the aircraft described in this paper is qualitatively very similar to the one with rigid wings. ${ }^{2}$ Instead, turning flight presents several instructive distinctions.

Wing twist can be used anti-symmetrically on the two wings to generate rolling moment, and differential dihedral can serve as a yaw control mechanism as explained in Sec. III. The steady state turn rate is an important metric for evaluating the maneuverability of an aircraft. In order to study the steady state turning performance of the aircraft, the equilibria corresponding to a steady turn are computed. In order to analyse a turning maneuver, the requisite constraint equations are added to the ordinary differential equations and the corresponding control parameters are "freed," i.e., treated as de facto state variables. The enlarged set of equations is then solved to compute the aircraft trim states and control inputs needed to achieve those trims. While this procedure yields the desired equilibria, it does not yield other equilibria which may exist for the given control inputs and do not satisfy the aforementioned constraints. ${ }^{21}$

Figures 6(a) and 6(b) plot the turn rate and the differential dihedral required for regulating sideslip, respectively, as functions of the wing twist during a coordinated turn. During this maneuver, the speed is held constant at $V=1.7 \mathrm{~m} / \mathrm{s}$. From a performance perspective, it is observed that a substantial amount of asymmetric (not necessarily anti-symmetric) dihedral is needed to maintain zero sideslip. The dihedral required for regulating the sideslip increases rapidly as the turn rate is increased above zero. Thereafter, the dihedral angles on both wings settle down to near-constant values even as the turn rate increases.

Figure $6(\mathrm{~b})$ also shows that $\delta_{R} \approx-\delta_{L}$ for trim at small values of wing twist. This is equivalent to an aircraft banking to one side while performing a turn. This is observed for small values of turn rate, upto $10 \mathrm{deg} / \mathrm{s}$. The corresponding pitch rate and fuselage bank angle are both small. As the turn rate builds up, so does the pitch rate as the aircraft bank angle increases, which leads to a deviation from this simple 


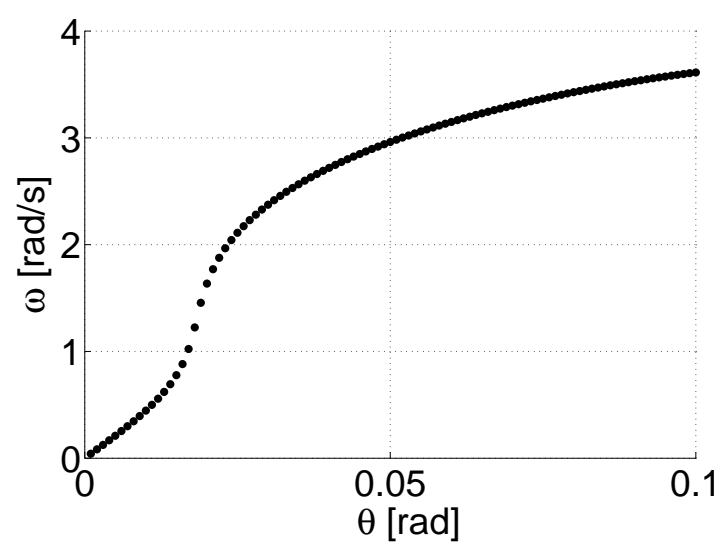

(a) Turn rate as a function of wing twist with the constraint $\beta=0$.

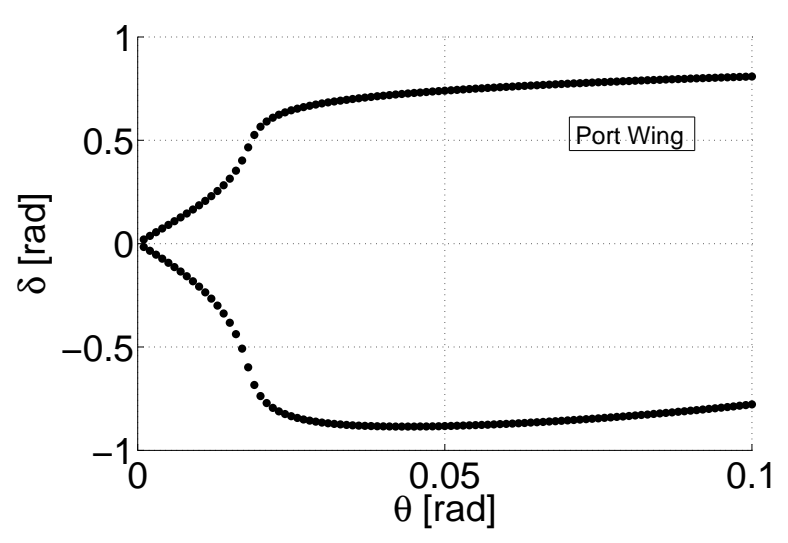

(b) Wing dihedral required to maintain $\beta=0$.

Figure 6. Coordinated turn trims with a fixed velocity $V=1.7 \mathrm{~m} / \mathrm{s}$. The equilibria are marked with filled circles, which indicates that they are all stable. The Young's modulus is $E=50 \mathrm{MPa}$.

expression.

A comparison with the corresponding plots for a rigid aircraft in Ref. [2] shows that flexibility does not improve the performance in a substantial way. Yet, it manifests itself in a most peculiar way. Whereas the outer wing has a larger dihedral in a rigid setting, the inner wing requires a larger dihedral at the wing root in the presence of flexibility. This is because of the fact that the wing possesses a natural tendency to settle down to a positive dihedral angle on account of the fact that it produces a positive lift. Therefore, when the wing is required to produce a negative dihedral effect (in the sense of an outward-pointing side force), this tendency has to be compensated by setting a larger anhedral at the wing root. This may be viewed as a detrimental effect of wing flexibility. Interestingly enough, axial tension in the wing can be fruitfully employed to stiffen the wing in such situations.

Although stability is not addressed in this paper, the peculiar topology in Fig. 6(a) suggests the possibility of an impending instability. It is quite possible that the equilibrium curve may fold over twice in the region between $\theta=0$ and $\theta=0.2 \mathrm{rad}$ if the values of the other control parameters are chosen differently. In that case, the aircrft would demonstrate jump-instabilities and hysteresis. ${ }^{23}$ Such a topology is indeed observed when $E$ is reduced to $5 \mathrm{MPa}$, as shown in Fig. 7(a) and 7(b). In such cases, axial tension can also be scheduled cleverly as a function of wing twist to mitigate the susceptibility of the aircraft to jumps.

Figure 8(a) shows the turn rate as a function of wing twist when the wing twist is fixed at $5 \mathrm{~g}$., while Fig. 8(b) plots the wing dihedral required to maintain zero sideslip. Clearly, and consistent with predictions based on Fig. 5(a), the dihedral deflection on the right wing (the wing under tension) is actually greater in magnitude than when it is not under any axial tension. However, there is no longer a jump like the one seen in Figs. 7(a) and 7(b). Such studies need to be performed on a case-by-case basis in the absence of a concrete theoretical explanation.

\section{Conclusions}

This paper described the steady state performance of an agile MAV equipped with flexible wings whose dihedral and twist are used to control the lateral - directional motion of the aircraft. A novel actuation scheme, in the form of wing tension, was considered in this paper. A complete nonlinear flight dynamic model was derived incorporating the flexible dynamics of the wing and variable center of gravity location. The concept of effective dihedral was introduced, and the role of wing dihedral in regulating sideslip during turning flight was demonstrated. It was shown that wing flexibility increases the anhedral required on the inner wing. It was shown that tension reduces the dihedral as well as the anhedral effects of the wing. While this seems to limit its intuitively suggestive utility as an actuator for turning flight, there is a need to investigate this matter further using a nonlinear structural deformation theory. Future work will focus on 


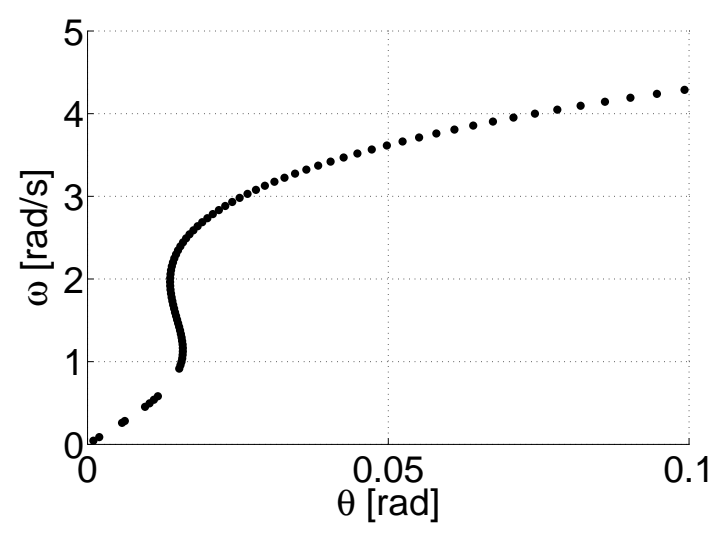

(a) Turn rate as a function of wing twist with the constraint $\beta=0$.

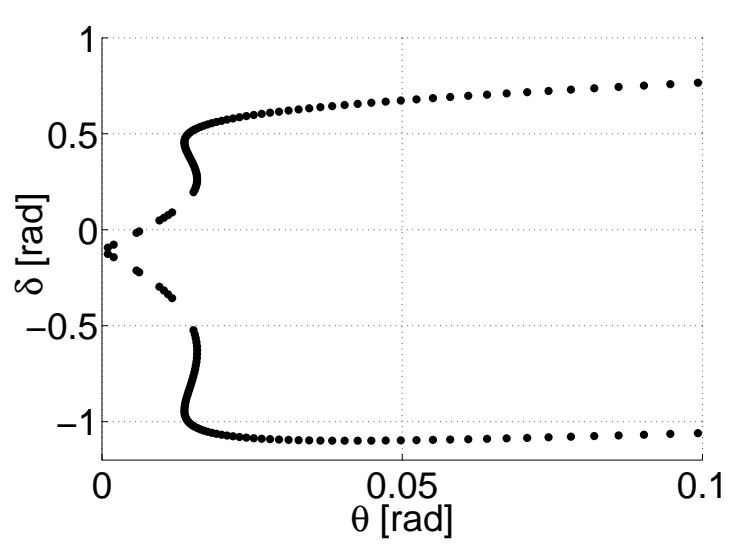

(b) Wing dihedral required to maintain $\beta=0$.

Figure 7. Coordinated turn trims with a fixed velocity $V=1.7 \mathrm{~m} / \mathrm{s}$. The equilibria are marked with filled circles, which indicates that they are all stable. The Young's modulus is $E=5 \mathrm{MPa}$.

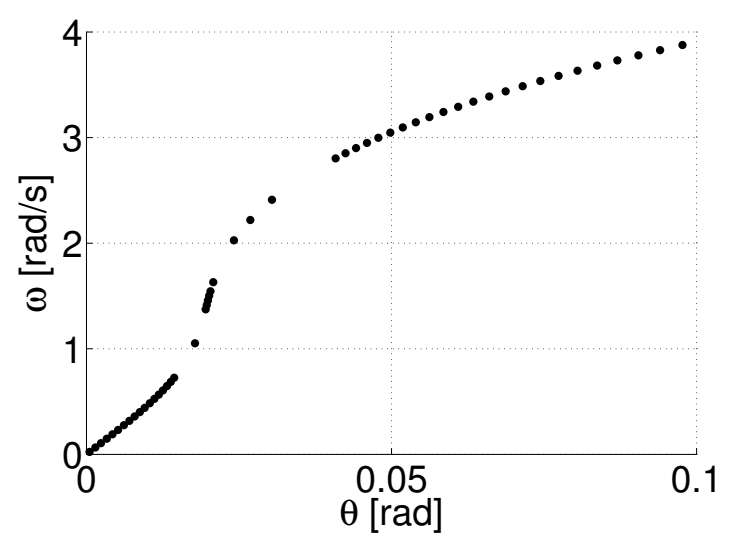

(a) Turn rate as a function of wing twist with the constraint $\beta=0$.

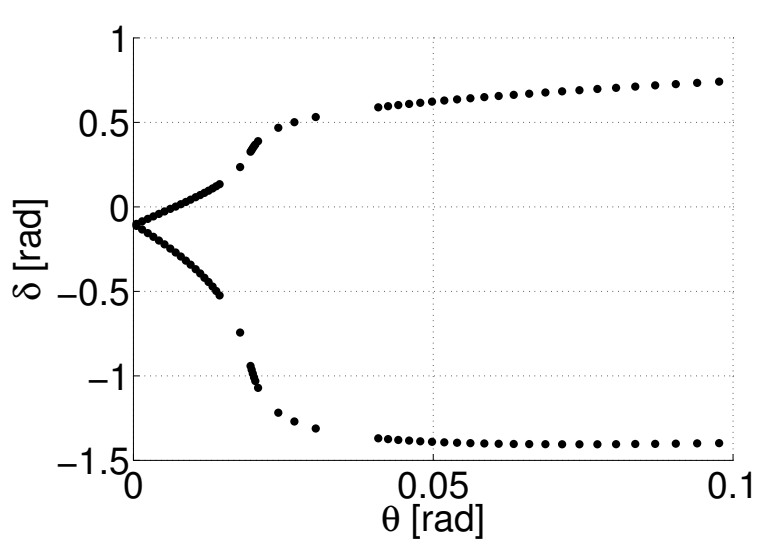

(b) Wing dihedral required to maintain $\beta=0$.

Figure 8. Coordinated turn trims with a fixed velocity $V=1.7 \mathrm{~m} / \mathrm{s}$ and the wing tension set to 5 g. The equilibria are marked with filled circles, which indicates that they are all stable. The Young's modulus is $E=5 \mathrm{MPa}$.

improving the structural model of the wing, completing the stability calculations and exploring the role of wing tension using large deformation models.

\section{Acknowledgments}

This project was supported by the Air Force Office of Scientific Research (AFOSR) under the Young Investigator Award Program (Grant No. FA95500910089) monitored by Dr. W. Larkin. The original problem was posed by Dr. Gregg Abate (AFRL). This paper also benefitted from stimulating discussions with Mr. Johnny Evers (AFRL). 


\section{References}

${ }^{1}$ Ol, M., Parker, G., Abate, G., and Evers, J., "Flight Controls and Performance Challenges for MAVs in Complex Environments," AIAA Guidance, Navigation and Control Conference and Exhibit, 2008. AIAA Paper 2008 - 6508.

${ }^{2}$ Paranjape, A. A., and Chung, S.-J., "Flight Mechanics of a Tail-less Articulated Wing Aircraft," Proc. AIAA Atmospheric Flight Mechanics Conference, Toronto, CA, AIAA Paper 2010 - 7633, 2010.

${ }^{3}$ Mueller, T. J., Fixed and Flapping Wing Aerodynamics for Micro Air Vehicle Applications, Progress in Aeronautics and Astronautics, Vol. 195, AIAA, 2001.

${ }^{4}$ Costello, M., and Webb, A., "Wing Articulation of Micro Air Vehicles to Reduce Gust Sensitivity," AIAA Paper 2008 $6712,2008$.

${ }^{5}$ Wickenheiser, A., and Garcia, E., "Longitudinal Dynamics of a Perching Aircraft," Journal of Aircraft Vol. 43, No. 5, 2006, pp. $1386-1392$.

${ }^{6}$ Wickenheiser, A., and Garcia, E., "Optimization of Perching Maneuvers through Vehicle Morphing," Journal of Guidance, Control and Dynamics, Vol. 31, No. 4, 2008, pp. 815 - 823.

${ }^{7}$ Reich, G. W., Wojnar, O., and Albertani, R., "Aerodynamic Performance of a Notional Perching MAV Design," AIAA Paper 2009 - 63, 2009.

${ }^{8}$ Sachs, G., and Holzapfel, F., "Flight Mechanic and Aerodynamic Aspects of Extremely Large Dihedral in Birds," AIAA Paper 2007 - 46, 2007.

${ }^{9}$ Sachs, G., "What Can Be Learned From Unique Lateral-Directional Dynamics Properties of Birds for Mini-Aircraft," AIAA 2007-6311, 2007.

${ }^{10}$ Sachs, G., "Why Birds and Miniscale Airplanes Need No Vertical Tail," Journal of Aircraft, Vol. 44, No. 4, 2007, pp. 1159 $-1167$.

${ }^{11}$ Paranjape, A. A., and Chung, S.-J., "Lateral Stability of Vertical Tailless Aircraft with Variable Wing Dihedral," under review, Journal of Guidance, Control and Dynamics, 2010.

${ }^{12}$ Waszak, M. R., and Schmidt, D. K., "Flight Dynamics of Aeroelastic Vehicles," Journal of Aircraft, Vol. 25, No. 6, 1988, pp. 563 - 571.

${ }^{13}$ Meirovitch, L., and Tuzcu, I., "Unified Theory for the Dynamics and Control of Maneuvering Flexible Aircraft," AIAA Journal, Vol. 42, No. 4, 2004, pp. 714 - 727.

${ }^{14}$ Nguyen, N., and Tuzcu, I., "Flight Dynamics of Flexible Aircraft with Aeroelastic and Inertial Force Interactions," Proc. AIAA Atmospheric Flight Mechanics Conference, Chicago, IL., 2009, AIAA Paper 2009 - 6045.

${ }^{15}$ Patil, M. J., and Hodges, D. H., "Flight Dynamics of Highly Flexible Flying Wings," Journal of Aircraft, Vol. 43, No. 6, 2006, pp. $1790-1798$.

${ }^{16}$ Raghavan, B., and Patil, M. J., "Flight Dynamics of High-Aspect-Ratio Flying Wings: Effect of Large Trim Deformation," Journal of Aircraft, Vol. 46, No. 5, 2009, pp. 1808 - 1812.

${ }^{17}$ Baghdadi, N., Lowenberg, M. H., and Isikveren, A. T., "Application of Bifurcation and Continuation Methods to Nonlinear Flexible Aircraft Dynamics," Proc. AIAA Guidance, Navigation and Control Conference 2009, Chicago, IL. AIAA Paper $2009-6305$.

${ }^{18}$ Chung, S.-J., and Dorothy, M., "Neurobiologically Inspired Control of Engineered Flapping Flight," Journal of Guidance, Control and Dynamics, Vol. 33, No. 2, 2010, pp. 440 - 453.

${ }^{19}$ Dickinson, M. H., Lehmann, F. -O., and Sane, S. P., "Wing Rotation and the Aerodynamic Basis of Insect Flight," Science, Vol. 284, 1999, pp. $1954-1960$.

${ }^{20}$ Shampine, L. F., Reichelt, M. W., and Kierzenka, J., "Solving Boundary Value Problems for Ordinary Differential Equations with bvp4c," available at http : //www.mathworks.com/bvp_tutorial.

${ }^{21}$ Paranjape, A. A., Sinha, N. K., and Ananthkrishnan, N., "Use of Bifurcation and Continuation Methods for Aircraft Trim and Stability Analysis - A State-of-the-Art" Journal of Aerospace Sciences and Technologies, Vol. 60, No. 2, 2008, pp. 1-12. Abridged version: AIAA Paper 2007 - 1051.

${ }^{22}$ Stanford, B., Abdulrahim, M., Lind, R., and Ifju, P., "Investigation of Membrane Actuation for the Roll Control of a Micro Air Vehicle," Journal of Aircraft, Vol. 44, No. 3, 2007, pp. 741 - 749.

${ }^{23}$ Mahale, A., and Ananthkrishnan, N., "Analytical Criterion for Onset of Departure in Inertia-Coupled Roll Maneuvers of Airplanes," AIAA Paper 2006-6267, 2006. 